\title{
Application of the extended poroacoustic model of marine sediments to the calculation of the sound reflection coefficient from the seabed
}

\author{
$V$ Lisyutin $^{1, *}, A$ Yaroshenko ${ }^{1}, O$ Lastovenko $^{1}$ \\ ${ }^{1}$ Sevastopol State University, Universitetskaya 33, Sevastopol 299053, Russia
}

\begin{abstract}
The article consider the application of the extended poroacoustic theory of the propagation of stress waves in marine sediments to the calculation of the coefficient of sound reflection from the seabed. It is proposed to calculate the reflection coefficient using a simple acoustic formula, replacing the equilibrium density of the porous medium in it with the effective density. The main assumptions are given and the dispersion relations of the extended poroacoustic model of unconsolidated marine sediments are derived (GSED). Model GSED allows calculating the frequency dependences of the propagation velocity and the attenuation coefficients of sound and shear waves in marine sediments. Internal friction between granules and viscous losses due to relative fluid movement are taken into account. The adequacy of GSED model is shown by comparison with experimental data taken from open sources. The results of calculating the reflection coefficient within the framework of the proposed method are compared with experimental data.
\end{abstract}

\section{Introduction}

There is an increasing need for shallow water acoustics to fully understand the interaction of acoustic waves with the seabed composed of unconsolidated marine sediments. The plane wave reflection coefficient from the seabed is particularly important in underwater acoustics. Once the reflection coefficient is known, realistic propagation modeling can assist in communication, detection, and classification in coastal environments [1]. Acoustic profiling is an effective method for researches the seabed and marine sediments. In this case, the reflection coefficient is determined at a normal angle of incidence [2,3]. More complex, but also more informative methods require calculating the frequency and angle dependences of the reflection coefficient [4]. Remote sensing of bottom sediments requires a theoretical model to estimate the parameters of bottom sediments and sediments classification [5].

Marine sediments have been characterized by five parameters: the compressional ( $p$ wave) and shear ( $s$-wave) wave velocities $c_{p}, c_{s}$, attenuation coefficients $\alpha_{p}, \alpha_{s}(\mathrm{~Np} / \mathrm{m})$, their frequency $(f, \mathrm{~Hz})$ dependences, and density.

\footnotetext{
* Corresponding author: vlisiutin@mail.ru
} 
To calculate the reflection coefficient, the wave numbers stress waves are represented as

$$
k_{p, s}=\omega / c_{p, s}-\mathrm{i} \alpha_{p, s}=\omega / c_{p, s}\left(1-\mathrm{i} \beta_{p, s}\right),
$$

where $\beta_{p, s}=\alpha_{p, s} c_{p, s} / \omega$ is the loss tangent, $\omega=2 \pi f, \mathrm{i}=\sqrt{-1}$.

The speed of the sound wave mainly depends on the porosity, the speed of the shear wave is in the range of $80 \ldots 150 \mathrm{~m} / \mathrm{s}$, but does not affect the normal reflection coefficient. A significant uncertainty in bottom losses is introduced by the frequency dependence of the attenuation coefficient [2-4].

In dry granular media, as established experimentally, the attenuation coefficient $(\alpha$, $\mathrm{dB} / \mathrm{m}=8.69 \alpha, \mathrm{Np} / \mathrm{m})$ is proportional to the first power of frequency, $\alpha_{\mathrm{p}, \mathrm{s}}=\alpha_{\mathrm{p}, \mathrm{s} 0} f^{1}$, $\left(\alpha_{p, s 0}=2 \pi \cdot 8,69 \cdot \beta_{p, s} / c_{p, s}\right.$ is the attenuation coefficient pertaining to the frequency unit, $\mathrm{dB} / \mathrm{m} / \mathrm{Hz}$ ). Measurements in water-saturated media confirm the proportionality of the attenuation coefficient $\alpha \sim \omega^{1}$ for low-permeable media, but deviations from the law $\alpha \sim \omega^{1}$ have been detected for highly permeable media [6]. The complex frequency dependence of attenuation presupposes at least two physical loss mechanisms. The main, inherent to both dry and saturated media, is called internal friction, and the second, viscous dissipation during pore fluid movement with respect to granules. Internal friction yields a directly proportional dependence; viscous loss, deviations from direct proportionality in a certain frequency range [6].

\section{Calculation of the reflection coefficient and bottom loss in the poroacoustic approximation}

Several plane wave models exist that are used to calculate the reflection coefficient given the sediment material parameters.

The most famous poro-elastic theory of stress-wave propagation in marine sediments is the Biot-Stoll theory [7]. In the Biot-Stoll theory, sediments are considered as a two-phase medium in which mineral grains are consolidated by an elastic skeletal frame and the pore fluid is mobile. Attenuation results from the fluid viscosity and internal friction. To account for internal friction, a small complex part is assigned to the elastic constants [7]. In this case, at the matching frequency, the ratio between the real and imaginary parts of the elastic moduli are true, while at high frequencies this is violated and viscous loss is proportional to $\omega^{1 / 2}$, which contradicts the experimental data. The Bio-Stoll theory includes 13 input parameters, and four boundary conditions are required to calculate the sound reflection coefficient from the bottom [7].

K.L. Williams developed model of "Effective Density Fluid" (EDFM) to calculate the reflection coefficient of sound from the bottom using simple acoustic formulas [8,9]. Based on the classical theory of Biot, the EDFM model is a reduced version of Biot's original formulation in which the frame bulk modulus and the shear modulus have been set to zero. Neglect of the elastic frame amounts to treating the porous medium as a homogeneous fluid, capable of supporting Biot's "fast" compressional wave but not the "slow" wave nor the shear wave of the full Biot theory. The EDFM theory shows incorrect attenuation - at high frequencies $\alpha \sim \omega^{1 / 2}$. Despite its incorrectness, EDFM theory proved to be in efficient and demand [9-13].

M. Buckingham developed the GS (Grain Shearing) and VGS (Viscous Grain Shearing) theories, which explain the propagation and attenuation of strain waves in sediments owing to intergranular friction [14, 15]. Friction imparts the medium with longitudinal and 
transverse rigidity, but at the same time causes energy losses. In GS theory, sediments are treated as a single-phase medium and viscous losses are not taken into account. The frequency dependence of the attenuation coefficient is $\alpha \sim \omega^{1}$, which corresponds to experiments only at high frequencies [14]. In VGS theory, viscous loss is "simulated" by a fluidlike rheological element [15]. Simulation of viscous losses prevents establishing a connection between the acoustic properties of stress-waves and the physical properties of the medium.

The works $[16,17]$ present the theories of GSEC, based on the Grain Shearing theory. According to the GS + Effective Compressibility model, the bulk modulus of sediments is assumed to be complex and frequency-dependent. The fluid mobility to flow from the gap between the granules increases the compressibility of the medium at low frequencies. On the other hand, as the frequency increases, the fluid viscosity appears, preventing it from being squeezed out of the gap into a more capacious part of the pore space, which leads to an increase in the medium rigidity. Due to an increase in the real part of the bulk modulus of sediments, dispersion of the speed of sound occurs. The imaginary part of the bulk modulus determines the viscous loss.

In this work, we propose an extended poroacoustic model of marine sediments, which assumes the possibility of propagation of two types of waves, fast compressional and shear waves.

The poroacoustic reflection coefficient model is a function of the incident angle and the characteristic acoustic impedances of the two mediums [9]. The poroacoustic model includes an additional impedance for the shear wave propagation. For the case

$$
R=\frac{Z_{t o t}-Z_{f}}{Z_{t o t}-Z_{f}}
$$

where $Z_{f}=\frac{\rho_{f} c_{f}}{\omega \cos \theta_{i}}, Z_{t o t}=Z_{p} \cos ^{2} 2 \chi_{s}+Z_{s} \sin ^{2} 2 \chi_{s}$,

$$
Z_{p}=\frac{\rho_{2 p}}{\left(k_{p}^{2}-k_{f}^{2} \sin ^{2} \theta_{i}\right)^{1 / 2}}, Z_{s}=\frac{\rho_{2 s}}{\left(k_{s}^{2}-k_{f}^{2} \sin ^{2} \theta_{i}\right)^{1 / 2}}
$$

In the above equations, $R$ is the reflection coefficient and $Z_{f}$ is the impedance of the water column. $Z_{\text {tot }}$ is the total impedance of the sediment and $Z_{p}$ is the impedance of the compressional wave while $k_{p}$ is the complex compressional wavenumber. $Z_{s}$ is the impedance of the shear wave while $k_{s}$ is the complex shear wave number and the incident angle, $\theta_{i}$. The transmission angle of the shear wave, $\chi_{s}$ can be determined using Snell's law.

We pay special attention to the density $\rho_{2 p}$ and $\rho_{2 s}$ in the equation (3). The poroacoustic model is based on simple acoustic boundary conditions of contact between two liquid media. However, porous fluid-saturated unconsolidated marine sediments are not fluid. Therefore, the quantities $\rho_{2 p}$ and $\rho_{2 s}$ are not the equilibrium densities of the two-component medium, but the effective densities of the two-phase medium included in the dispersion relations for the complex phase velocities of the compression and shear waves. Effective densities will be determined below. This is the fundamental difference between our model and the one that was used, for example, in our work [9].

Bottom loss is defined as $B L=-20 \log _{10}|R|$, dB. 


\section{Extended poroacoustic model of unconsolidated water- saturated marine sediments}

According to GS theory, the complex phase velocities $\tilde{c}_{p, s}=\omega / k_{p, s}$ of compressional and shear waves can be represented as [14-17]

$$
\tilde{c}_{p}=\sqrt{\frac{K_{0}+\gamma\left(\mathrm{i} \omega t_{0}\right)^{n}}{\rho_{0}}}, \tilde{c}_{s}=\sqrt{\frac{\gamma_{s}\left(\mathrm{i} \omega t_{0}\right)^{m}}{\rho_{0}}},
$$

where $K_{0}=\left(\frac{P}{K_{f}}+\frac{1-P}{K_{g}}\right)^{-1}-$ bulk modulus of the medium, $P$ - porosity, $K_{f}, K_{g}$ are the elastic moduli of the fluid and solid phase; $\rho_{0}=P \rho_{f}+(1-P) \rho_{g}$ - is the equilibrium density of the medium, $\rho_{f}, \rho_{g}$ are density of the fluid and solid phase; $\gamma, \gamma_{\mathrm{s}}$ is the composite and shear intergranular rigidity, $\mathrm{Pa} ; n, m$ is the compressional and shear strain hardening exponent, reflecting the magnitude of nonlinearity of the medium, $0<n<1,0<m<1$; $t_{0}=$ $1 \mathrm{~s}$ is the formal constant, which restoring the correct physical dimension. Four parameters, $\gamma, \gamma_{\mathrm{s}}, n$, and $m$ characterize the intergranular interaction at the microlevel and can only be determined by inverting the experimental data $[16,17]$.

The real velocities $c_{p, s}$ and attenuation coefficients $\alpha_{p, s}$ can be obtained from the complex phase velocities as $c_{p, s}=\left(\operatorname{Re}\left(\tilde{c}_{p, s}^{-1}\right)\right)^{-1}, \alpha_{p, s}=-\omega \operatorname{Im}\left(\tilde{c}_{p, s}^{-1}\right), \mathrm{Np} / \mathrm{m}$.

Note that dispersion relations (4) take into account only internal friction. To transform one-phase equations (4) into quasi-two-phase (i.e., in the poroacoustic approximation), it is necessary to join the effects caused by the interaction of solid and fluid phases during their relative motion. The simplest way is to replace the equilibrium density in Eq. (4) by the complex effective density $[8,18]$.

Compressional wave. The equation for the effective density (Eq. (14), Ref. [8]) in the EDFM theory is derived assuming a global Biot flow in a porous medium [8]. The intensity of the global flow is related to the permeability of the medium.

However, the pore space of a real medium has a network structure consisting of seriesparallel connected segments with different cross sections. Inside wide pores, there are small particles between large granules that narrow or even clog the flow area. In such a medium, both global and local flows are possible [17]. The fraction of the bulk of the medium in which a flow, global other local, is possible is called the effective porosity and denoted $\phi$ [17]. Since local flows are not directly related to the permeability of the medium, the permeability should be excluded from the equation for the effective density using the relation $\phi=8 T \kappa_{0} / a^{2}$, where $\kappa_{0}$ is the static (steady-state) permeability of the medium, $\mathrm{m}^{2} ; T$ - tortuosity; $a$ - pore radius or pore size parameter, $\mathrm{m}$ (model pores are assumed to be cylindrical pipes).

In real marine sediments, the ratio $K_{0} \gg \gamma$ [17], is always fulfilled, which makes it possible to neglect internal friction and represent the medium as a suspension. Let us calculate the effective density of such a fluid-like medium, assuming in the Biot equations of motion the elastic constants of the skeletal frame to be equal to zero. Repeating transformations Ref. [8], after some algebra, we obtain an expression alternative to Eq. 14, Ref [8] for the effective density of the medium 


$$
\rho_{p e f f}=\frac{T \rho_{f} \rho_{0}-\phi \rho_{f}^{2} F_{C}(w)}{T \rho_{f}+\phi\left(\rho_{0}-2 \rho_{f}\right) F_{C}(w)},
$$

where $F_{C}(w)=1-\frac{2 J_{1}\left(\mathrm{i}^{3 / 2} w\right)}{\mathrm{i}^{3 / 2} w J_{0}\left(\mathrm{i}^{3 / 2} w\right)}-$ the frequency correction function;

$$
w=a \sqrt{\omega \rho_{f} / \eta},
$$

$J_{0.1}$ are Bessel functions, and $\eta$ is the dynamic fluid viscosity, $\mathrm{Pa} \cdot \mathrm{s}$. The frequency correction function reflects the change in the nature of the flow in the pores with increasing frequency.

Replacing the equilibrium density in the first Eq. (4) by the effective density (5), we obtain the dispersion relation for the compressional wave in the poroacoustic approximation. We will call this model GSEDp.

Shear wave. The propagation of a shear wave in an unconsolidated medium is possible only due to the forces of intergranular friction. Comparison of the second Eq. (4) with the dispersion relation for the shear wave of the Biot-Stoll theory [7], after simplifications, leads to the following expression for the shear effective density

$$
\rho_{\text {seff }}=\rho_{0}-T_{s}^{-1} \phi \rho_{f} F_{C}(w),
$$

Another way to derive Eq (7) is given in the work [18]. Replacing the equilibrium density in the second Eq. (4) by the effective density (7), we obtain the dispersion relation for the shear wave in the extended poroacoustic approximation. We will call this model GSEDs. To calculate the reflection coefficient in equations (3), we must put $\rho_{2 p}=\rho_{p e f f}(5)$ and $\rho_{2 s}=$ $\rho_{\text {seff }}(7)$.

\section{Comparison of the results of poroacoustics models with experimental data}

The input parameters determining the sound speed and attenuation are the composite intergranular rigidity $\gamma$ and stress relaxation exponent $n$, respectively. The slope, shape of the dispersion curve, deviation of the attenuation coefficient from the law $\alpha_{p} \sim f^{1}$ is determined by the effective porosity $\phi-$ an internal parameter of the GSED model that relates acoustic and physical properties of the medium.

It is difficult to find an exact definition for the characteristic pore size. For a compression wave, Ref. [17] gives the estimate: $a=d_{e} / 7,35=0,136 d_{e}$, where $d_{e}=39 \sqrt{\kappa_{0}}$ are equivalent diameter of the spherical beads, i.e. having the same permeability as the real medium.

The measurement results that have the greatest practical significance for the acoustics of the shallow water are analyzed below. The results were obtained during the SAX-99 marine experiment (Sediment Acoustics Experiment) [15,17,19]. The phase velocities and attenuation of the comressional wave were measured on site by various systems at different frequencies. Common input parameters are as follows $d=0,379 \mathrm{~mm}$ - average diameter of sand granules; $\rho_{g}=2690 \mathrm{~kg} / \mathrm{m}^{3} ; K_{\mathrm{g}}=3,2 \cdot 10^{10} \mathrm{~Pa} ; \rho_{f}=1023 \mathrm{~kg} / \mathrm{m}^{3} ; K_{f}=2,395 \cdot 10^{9} \mathrm{~Pa} ; \eta=1$ $\mathrm{mPa} \cdot \mathrm{s} ; P=0,372 ; \kappa_{0}=2,5 \cdot 10^{-11} \mathrm{~m}^{2} ; T=1,35$. 
Experimental points and frequency dependences of the sound speed and attenuation coefficients are shown in Figure 1. It also shows other measurements of the speed of sound made at the point SAX-99. Data taken from work [20]. As can be seen from Figure 1, their speed of sound is lower.

The inversion results are as follows: $a=24 \mu \mathrm{m} ; K_{0}=5,7 \cdot 10^{9} \mathrm{~Pa} ; \gamma=2,1 \cdot 10^{7} \mathrm{~Pa} ; n=$ 0,$19 ; \phi=0,23$. The lower curve on the sound speed graph is plotted with the indicated intergranular friction parameters, but with higher porosity, $P=0,4$.
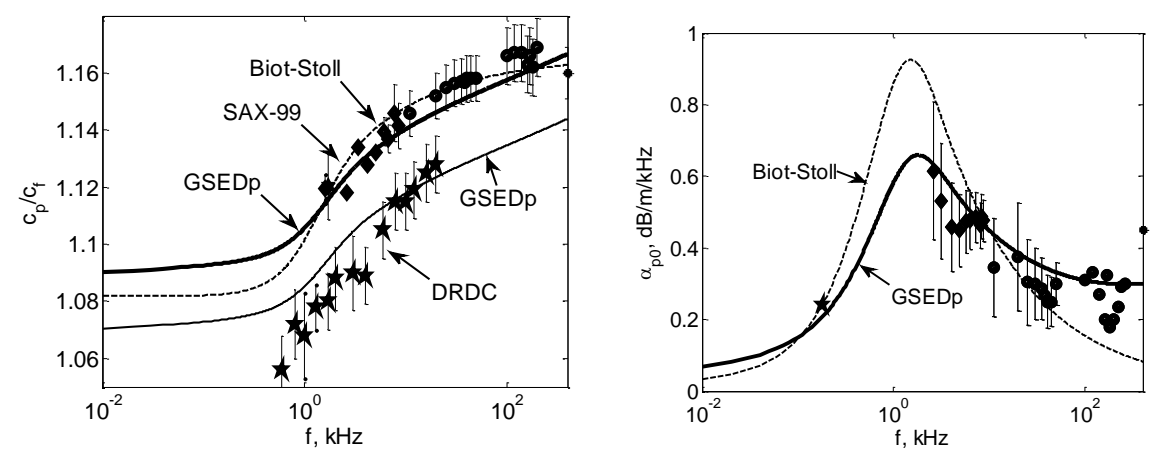

Fig. 1. Frequency dependent of sound speed and attenuation coefficient, $\mathrm{dB} / \mathrm{m} / \mathrm{kHz}$.

Figure 1 also shows the dispersion and dissipation curves of the Biot-Stoll theory. As you can see, at low frequencies, the Biot-Stoll theory shows a lower speed of sound, at medium frequencies - overestimated attenuation, and at high frequencies - underestimated attenuation. To plot the speed of sound, the porosity was taken equal to $P=0,385$.

Since $K_{0} \gg \gamma$, the speed of sound is determined mainly by the porosity of the medium, internal friction affects only slightly. On the contrary, the attenuation coefficient is determined by the internal friction and the degree of nonlinearity of the medium - the strain hardening exponent. The fluid movement and respectively the viscous loss manifests itself in the region of the middle frequencies, increasing the attenuation coefficient from 0,3 $\mathrm{dB} / \mathrm{m} / \mathrm{kHz}$ to $0,6 \mathrm{~dB} / \mathrm{m} / \mathrm{kHz}$.

Let us now consider the results of measurements of the reflection coefficient. According to [2], the bottom loss is $B L=-9,00 \pm 0,12 \mathrm{~dB}$ are $|R|=0,355 \pm 0,0052$. The results of calculating the frequency dependence of the reflection coefficient within the poroacoustic approximation are shown in Figure 2. As can be seen from the Figure 2, the reflection coefficient is very sensitive to the porosity value. Comparison of Figure 1 and Figure 2 shows that the inversion of the speed of sound, carried out by measuring the reflection coefficient, gives a result closer to the direct measurements marked "DRDC". Uncertainty of the porosity value of the same type of marine sediments, measured practically in the same point, can be $\pm 0,2$, which leads to a change in the bottom loss by $\pm 0,5 \mathrm{~dB}$.

\section{Discussion and conclusion}

An extended version of the poroacoustic theory assumes the possibility of shear wave propagation in unconsolidated marine sediments. It is shown that the GSED theory correctly reproduces the frequency dependence of the velocity and attenuation coefficient of compressional wave. 

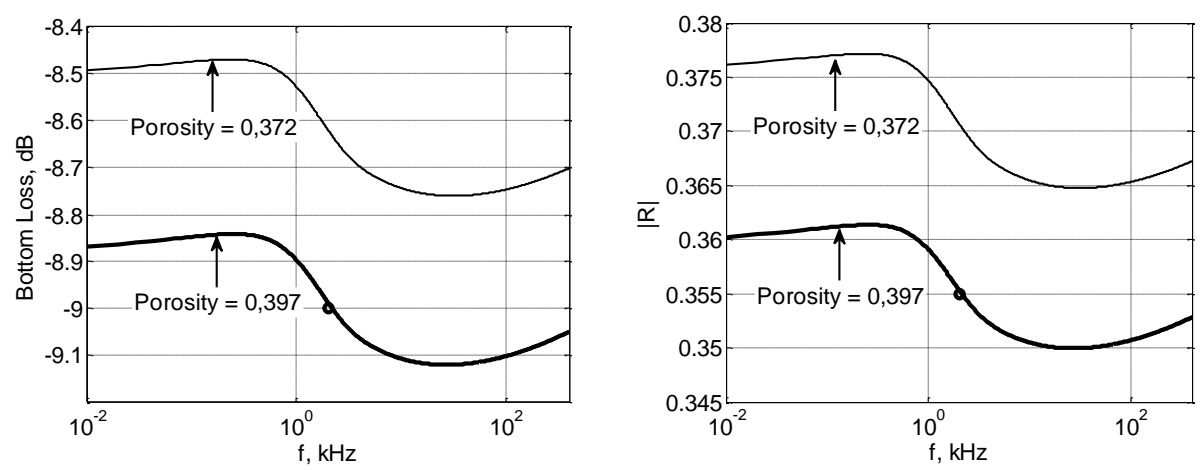

Fig. 2. Frequency dependence of the reflection coefficient and bottom loss.

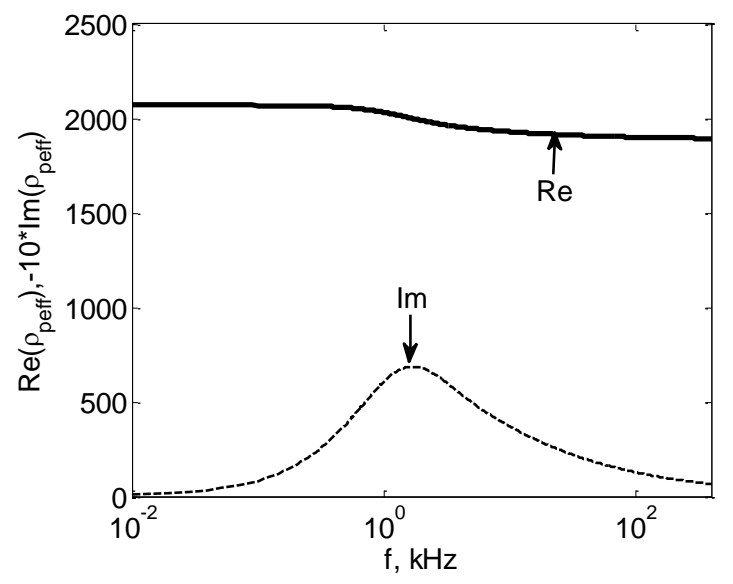

Fig. 3. Frequency dependence of the real and imaginary parts of the effective density.

The main purpose of the GSED model is to calculate of the sound reflection coefficient from the seabed when solving direct and inverse problems of shallow water acoustics. Simple acoustic boundary conditions lead to the need to correct the classical formula for the reflection coefficient of a sound wave from the interface between two liquid media. Instead of the equilibrium density, the effective density, which is different for sound and shear waves, should be substituted into formula (3).

Figure 3 shows the frequency dependence of the real and imaginary parts of the effective density. The imaginary part reflects the magnitude of viscous losses, i.e. proportional to the relative velocity between the two phases (solid and fluid), the real part reflects the inertial interaction and is proportional to the relative acceleration. At low frequencies, granules and fluid oscillate together, $\rho_{\text {peff }}=\rho_{0}$. At high frequencies, the granules and the part of the fluid, determined by the effective porosity, oscillate separately, but the viscous losses are small, $\rho_{\text {peff }}<\rho_{0}$. Viscous losses are maximum at medium frequencies.

Together with the speed of sound and the attenuation coefficient, the effective density has a complicated effect on the frequency dependence of the reflection coefficient. At low frequencies, the effective density is constant, the speed of sound increases and, accordingly, the reflection coefficient $(|\mathrm{R}|)$. At medium frequencies, the effective density decreases, and with it the reflection coefficient. At high frequencies, the effective density is constant, but 
the speed of sound increases due to internal friction. The reflection coefficient also increases.

\section{Acknowledgments}

The investigation is carried out within the framework of the SevSU internal grant "Development of theoretical models for physical methods of research of the Black Sea shelf", project No. 41/06-31.

\section{References}

1. Jackson D R and Richardson M D 2007 High-Frequency Seafloor Acoustics (New York: Springer) p 616

2. Schock S G 2004 IEEE J. of Ocean. Eng. $291200-17$

3. Chiu L Y C, Chang A, Lin Y-T and Liu C-S 2015 IEEE J. of Ocean. Eng. $40222-36$

4. Holland C W and Dettmer J 2013 J. Acoust. Soc. Am. 133 50-61

5. Rakotonarivo S, Legris M, Desmare R, Sessarego J-P and Bourillet J-F 2011 Geophysics 76 T91-T99

6. Kibblewhite A C 1989 J. Acoust. Soc. Am. 86 716-38

7. Stoll R D 1989 Sediment Acoustics (New York: Springer)

8. Williams K L 2001 J. Acoust. Soc. Am. 1102276 - 81

9. Camin H J and Isakson M J 2006 J. Acoust. Soc. Am. 1202437 - 49

10. Isakson M J, Neilsen T B 2006 J. Acoust. Soc. Am. 120135 - 44

11. Bonomo A L, Chotiros N P and Isakson M J 2015 J. Acoust. Soc. Am. 138748

12. Chotiros N P 2017 Acoustics of the Seabed as a Poroelastic Medium (Springer Briefs in Oceanography)

13. Buckingham M J 2000 J. Acoust. Soc. Am. 108 2796-2815

14. Buckingham M J 2007 J. Acoust. Soc. Am. 122 1486-1501

15. Lisyutin V A 2019 Physical Oceanography 26(1) 77-91 doi:10.22449/1573-160X2019-1-77-91

16. Lisyutin V A and Lastovenko O R 2020 Acoust.Phys. 66 401-15 doi.org/10.1134/S1063771020040065

17. Lisyutin V A 2018 Ekol. Vestn. Nauchn. Tsentr. Chernomorsk. Ekon. Sotrudnichestva 15(3) 39-51 doi:10.31429/vestnik-15-3-39-5

18. Williams K L, Darrell R J, Thorsos E I, Tang D and Schock S G 2002 IEEE J. of Ocean. Eng. $27413-28$

19. Hines P C, Osler J C, Scrutton J G E and Halloran L J S 2010 IEEE J. of Ocean. Eng. 35 502-15 